\title{
Association of statin therapy with incidence of Type 2 diabetes among US Veterans
}

\author{
Luc Djousse $^{1,2 *}$, Rebecca J. Song ${ }^{1,3}$, Kelly Cho ${ }^{1,2}$, J. Michael Gaziano ${ }^{1,2}$, David R. Gagnon ${ }^{1,4}$ \\ ${ }^{I}$ From the Massachusetts Veterans Epidemiology and Research Information Center (MAVERIC), Boston \\ Veterans Affairs Healthcare System, Boston, MA. \\ ${ }^{2}$ The Division of Aging, Brigham and Women's Hospital and Harvard Medical School, Boston, MA. \\ ${ }^{3}$ Department of Epidemiology, Boston University School of Public Health, Boston, MA. \\ ${ }^{4}$ Department of Biostatistics, Boston University School of Public Health, Boston, MA.
}

\begin{abstract}
Aims: While some but not all trial data have suggested an elevated risk of Type 2 diabetes with statin use, limited data are available on the relation of statin treatment with glycaemia and risk of Type 2 diabetes among Veterans. We examined whether statin use was associated with a higher incidence of Type 2 diabetes and secondarily, if statin use was associated with high plasma glucose.

Methods: Prospective analysis based on electronic health records of 3,390,799 US Veterans from 2000 to 2012. We used the Veteran Administration Corporate Data Warehouse to obtain information on random plasma glucose. Statin use was captured using the pharmacy database. Type 2 diabetes was defined as having at least one inpatient diagnosis or at least two outpatient diagnoses of Type 2 diabetes using International Classification of Disease version 9 codes 250.xx, or the use of hypoglycemic agents. We used multi-level derived propensity score and inverse probability weighting to address confounding by indication and Cox regression to estimate relative risk of Type 2 diabetes.

Results: The mean age was $62 \pm 11.9$ years; $93.3 \%$ were men and $82.7 \%$ were white. During a median followup of 3.0 years, 443,104 new cases of Type 2 diabetes occurred. Compared to no statin use, multivariable adjusted hazard ratio (95\% CI) for Type 2 diabetes was 1.21 (1.19-1.24) for low statin potency, 1.22 (1.211.23) for medium statin potency, and $1.34(1.32-1.36)$ for high statin potency ( $p$ linear trend <0.0001). In secondary analysis, statin use was not associated with higher plasma glucose.

Conclusions: Our data show a positive association between statin use and incidence of Type 2 diabetes among US Veterans.
\end{abstract}

Keywords: Statin, Type 2 diabetes, serum glucose, risk factors, epidemiology.

\section{Introduction}

Statins are widely used for treatment of dyslipidemia and subsequent prevention of cardiovascular diseases (CVD) among adults [1,2]. Besides elevated low-density lipoprotein cholesterol, Type 2 diabetes is another major risk factor for CVD [3-5]. With the growing epidemic of overweight/obesity in the United States [6,7], Type 2 diabetes has been on the rise and it is anticipated that 350 million Americans will be diagnosed with Type 2 diabetes by 2030 [8]. This underscores the need primary prevention of Type 2 diabetes and identification of novel risk factors for Type 2 diabetes.

Prior randomized trials of statins have suggested that statin therapy might increase the risk of Type 2 diabetes; However, such data have not been consistent across trials. While some randomized trials including the West of Scotland Coronary Prevention Study [9], the Heart
Protection Study ${ }^{10}$, the Anglo-Scandinavian cardiac Outcomes Trial-Lipid Lowering Arm (ASCOT-LLA) [11], and the LIPID trial [12], showed no statistically significant increased risk of Type 2 diabetes with statin; JUPITER trial [13], PROSPER [14], and NAVIGATOR [15], reported a modest increased risk of Type 2 diabetes when comparing statin to placebo.

Corresponding Author: Luc Djousse, Boston VA Healthcare System (MAVERIC),165 S Huntington Ave, Boston MA 02130 Email: Luc.Djousse@VA.GOV Received date: January 19, 2019; Accepted date: February 04, 2019; Published date: February 06, 2019. DOI: https://doi.org/10.31546/JCCCVT.1002 
A recent meta-analysis of randomized trials found $11 \%$ increased risk of Type 2 diabetes comparing statin to placebo [pooled $\mathrm{HR}=1.11$ (95\% confidence interval (CI): 1.1-1.2)] [16,17]; there was also evidence of doseresponse of statin on Type 2 diabetes risk. It is possible that treatment with statin might decrease insulin sensitivity as well as insulin secretion in a dosedependent manner. Nonetheless, several questions remained unanswered.

Since trial participants may differ from the general population and compliance to statin treatment may be higher in trial participants than among free living individuals in community settings, it is not well established whether observed elevated risk of Type 2 diabetes in clinical trials can be observed in large and long-term prospective studies. Furthermore, it is less clear whether statin treatment induces short- or longterm changes in plasma glucose concentrations in subjects free of Type 2 diabetes. The current project sought to test the hypothesis that statin use is associated with elevated plasma concentration of glucose over time and subsequent elevated risk of Type 2 diabetes among US Veterans.

\section{Research in Context}

What is already known about this subject?

- Statin use is highly prevalent for the prevention of cardiovascular disease

What is the key question?

- Is statin use associated with a higher risk of developing Type 2 diabetes among veterans?

What are the new findings?

- Statin use was associated with a 22 to $34 \%$ higher risk of developing Type 2 diabetes among veterans.

- The higher the potency of statin, the higher the risk of developing Type 2 diabetes

- Statin use did not influence blood sugar in this population

How might this impact on clinical practice in the foreseeable future?

- Clinicians might select the dose/potency of statin based on the individual risk for developing Type 2 diabetes

Abbreviations: CI: confidence interval, CVD: cardiovascular disease, HR: hazard ratio, ICD: International classification of disease, VA: Veterans Affairs.

\section{Research Design and Methods \\ Study population}

The study population was derived from the Veterans Affairs (VA) National Database from January 1, 2000 through December 31, 2012. Eligible Veterans met the following criteria: a) at least 30 years of age at the time of first statin prescription (referred to as index date) for statin users and diagnosis of dyslipidemia (total cholesterol of $200+\mathrm{mg} / \mathrm{dl}$ or LDL-cholesterol of $130+$ $\mathrm{mg} / \mathrm{dl}$ ) for non-users of statin as documented in their electronic health records (electronic heath record); b) free of Type 2 diabetes at the index date; c) no usage of insulin or oral hypoglycemic agents; d) at least two VA visits between 2000 and 2012; or e) diagnosis of cardiovascular disease among non-users of statin.

For analyses of changes in plasma glucose concentrations following statin initiation, we also required statin users to have $\geq 1$ random glucose and $\geq 1$ triglyceride measurements in the year preceding statin initiation and $\geq 2$ random glucose measurements within 12 months after statin initiation. For subjects with multiple glucose measurements in a day, we randomly selected one glucose measurement per 24 hours. Lastly, we required statin users to have their first VA visit at least one year prior to first statin prescription. We excluded subjects that were being treated with ezetimibe and subjects with first statin prescription less than one year after their first VA visit. After these exclusion criteria, we had a final sample of 3,390,799 subjects of whom $1,832,722$ were statin users.

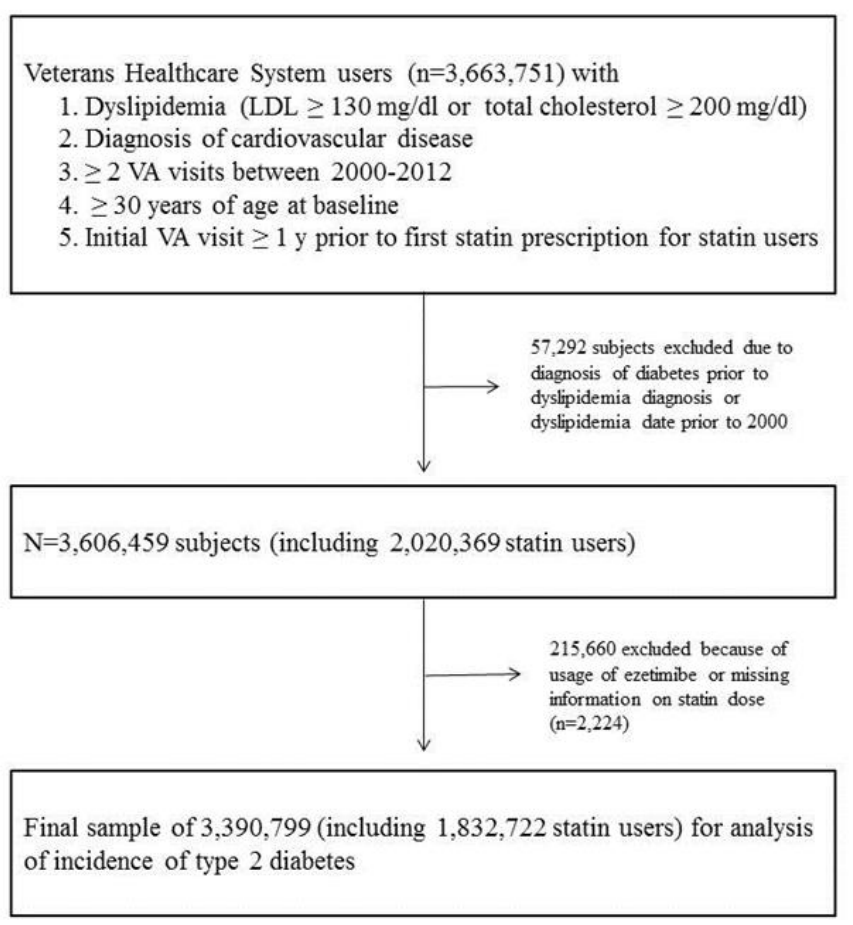

Figure-1 Flow Chart 


\begin{tabular}{|c|c|c|c|c|}
\hline \multirow[b]{2}{*}{ Characteristics } & \multirow[b]{2}{*}{ Non-user } & \multicolumn{3}{|c|}{ Statin users by potency } \\
\hline & & Low & Medium & High \\
\hline & $(\mathrm{n}=1,558,077)$ & $(\mathrm{n}=561,484)$ & $(\mathrm{n}=1,166,226)$ & $(\mathrm{n}=105,012)$ \\
\hline Age $(y)^{*}$ & $57(15)$ & $62(12)$ & $62(12)$ & $63(11)$ \\
\hline Male & $91 \%$ & $94 \%$ & $96 \%$ & $98 \%$ \\
\hline \multicolumn{5}{|l|}{ Race } \\
\hline White & $80 \%$ & $85 \%$ & $85 \%$ & $88 \%$ \\
\hline Black & $18 \%$ & $13 \%$ & $13 \%$ & $10 \%$ \\
\hline Other & $2 \%$ & $2 \%$ & $2 \%$ & $2 \%$ \\
\hline \multicolumn{5}{|l|}{ Ethnicity } \\
\hline Hispanic & $5.3 \%$ & $4.5 \%$ & $4.0 \%$ & $2.7 \%$ \\
\hline Body Mass Index $\left(\mathrm{kg} / \mathrm{m}^{2}\right)$ & $28.1(5.4)$ & $28.8(5.2)$ & $29.1(5.2)$ & $29.4(5.1)$ \\
\hline Total cholesterol $(\mathrm{mg} / \mathrm{dl})$ & $174.2(20.8)$ & $215.1(39.8)$ & $215(44.9)$ & $199.6(53.7)$ \\
\hline HDL cholesterol (mg/dl) & $47.3(15.4)$ & $45.3(13.4)$ & $44.9(13.1)$ & $44.1(13.0)$ \\
\hline LDL cholesterol (mg/dl) & $101.6(20.9)$ & $137.8(34.6)$ & $137.5(39.6)$ & $122.4(45.6)$ \\
\hline Triglycerides (mg/dl) & $130.7(92.1)$ & $169.6(135.6)$ & $175.4(145.1)$ & $178.0(170.2)$ \\
\hline $\begin{array}{l}\text { Systolic blood pressure } \\
(\mathrm{mmHg})\end{array}$ & $135.7(19.9)$ & $140.1(35.6)$ & $140.0(25.7)$ & $137.9(20.2)$ \\
\hline $\begin{array}{l}\text { Diastolic blood pressure } \\
\text { (mmHg) }\end{array}$ & $79.7(12.2)$ & $80.8(15.5)$ & 80.7 (14.7) & $79.4(12.2)$ \\
\hline
\end{tabular}

Age at dyslipidemia or first statin use; data presented as mean (SD) or percentage

Table-1: Baseline characteristics of 3,390,799 US Veterans according to statin prescription

\section{Assessment of index date for statin users}

Statin use was obtained using the VA prescription database. The index date was defined as the date of first statin prescription. Since statin brands vary by potency and dosage, we classified statin as low, medium, and high potency (supplemental Table 1 provides details).

\section{Assessment of random plasma glucose among veterans}

Plasma glucose measurements were obtained using laboratory data from the electronic health record in the VA database. When multiple glucose measurements were available on the same day, we randomly selected one measurement for current analyses. We did not use inpatient plasma glucose values taken on consecutive days to avoid acute and in-hospital treatment effect on glucose.

\section{Assessment of Type 2 diabetes}

Type 2 diabetes was defined as the presence of one inpatient ICD-9 code 250.xx or the presence of at least two outpatient ICD-9 codes 250 .xx for outpatients in the database or the initiation of either an oral or injectable diabetes mediation based on VA electronic prescription information and VA pharmacy fill information.

\section{Ascertainment of other variables}

Relevant covariates including age, gender, race, ethnicity, body mass index, blood pressure at index date, and date of birth were obtained from electronic heath record in the VA database. We also obtained information on comorbidity [cancer (except nonmelanoma skin cancer), chronic kidney disease, liver disease, thyroid disease, and heart disease]; laboratory parameters (lipids); and medications. Data from the Centers for Medicare \& Medicaid Services were used to enhance coverage and accuracy of the information abstracted from electronic heath record. Deaths were captured using Vital Status file in the VA Corporate Data Warehouse.

\section{Statistical Analysis}

Exploratory assessment of baseline glucose, triglycerides, cholesterol, body mass index, and other covariates was performed using means (standard deviation) for normally distributed variables and ranges, medians, and inter-quartile range for nonGaussian distributed variables. Graphical methods (e.g. histograms and boxplots) were used to visualize distribution of the data. We evaluated the association of statin use with plasma glucose by plotting daily mean glucose (95\% confidence intervals) during a period of 60 and 365 days pre- and post-initiation of statin therapy. In secondary analyses, we conducted stratified analyses by sex, triglycerides values ( $<$ and $\geq$ $150 \mathrm{mg} / \mathrm{dl}$ ), and statin potency (low, medium, and high). To address confounding by indication, we used multi-level propensity score (derived via logistic 
regression models that included predictors) to determine the probability of receiving no statin or low, medium, or high-potency statin. We then use these probabilities to weight study subjects using inverse probability of treatment weighting in the Cox regression model. We calculated person-time of follow up as time from index date to the first occurrence of a) Type 2 diabetes, b) death, c) prescription of ezetimibe during follow up, or d) end of follow-up period (December 31, 2012). To minimize immortal time bias, we consider the time between date of dyslipidemia and first prescription of statin as unexposed time for statin users. We calculated the incidence rate of Type 2 diabetes within each exposure group by dividing the number of new Type 2 diabetes events by the respective person-time of follow up. All analyses were performed using SAS Enterprise Guide (version 7.1). Alpha level was 0.05 and all tests were two-sided.

\section{Results}

Baseline characteristics of the $3,390,799$ subjects, the mean age was $62 \pm 11.9$ years [range: 18 to 105]; $93.3 \%$ were men, $82.7 \%$ were white, $15.1 \%$ African American, and $2.2 \%$ other race. The prevalence of statin use was $54.1 \%$ with predominance of simvastatin (83\%), followed by lovastatin (8\%) and pravastatin (4\%). Table- 1 presents characteristics of our study population.

\section{Association of statin use and incidence of Type 2 diabetes}

During a median follow-up of 3.0 years (range 0.003 to 12.9 years), we observed 443,104 new cases of Type 2 diabetes. There was a dose-response relation between statin potency and Type 2 diabetes risk; comparing to people that did not receive statin, hazard ratios (95\% CI) for Type 2 diabetes adjusted for age, sex, race, and body mass index were $1.21(1.19-1.24)$ for low, 1.22 (1.21$1.23)$ for medium, and 1.34 (1.32-1.36) for high potency statin, respectively ( $\mathrm{p}$ for linear trend $<0.0001$, Table 2 ). Similar results were observed in men [corresponding HR (95\% CI) of 1.17 (1.16-1.19), 1.25 (1.24-1.26), and 1.45 (1.42-1.48), respectively] and women [corresponding HR (95\% CI) of 1.36 (1.28-1.44), 1.58 (1.51-1.66), and 2.05 (1.78-2.35), respectively]. Furthermore, we did not observe an interaction between race or body mass index with statin use on the incidence of Type 2 diabetes (data not shown). Among those with triglycerides below 150 $\mathrm{mg} / \mathrm{dl}$ corresponding HRs (95\% CI) for low, medium, and high statin potency were 1.19 (1.17-1.21), 1.24 (1.22-1.26), and 1.43 (1.37, 1.50), respectively. Among Veterans with triglycerides $\geq 150 \mathrm{mg} / \mathrm{dl}$, corresponding HRs $(95 \%$ CI) were 1.14 (1.11 - 1.16), 1.20 (1.18 $1.22)$, and $1.38(1.33-1.43)$.

\begin{tabular}{|l|l|c|}
\hline & $\begin{array}{l}\text { Age-adjusted HR } \\
\text { (95\% CI) }\end{array}$ & $\begin{array}{l}\text { Multivariable } \\
\text { HR* }^{*} \mathbf{9 5 \%} \text { CI) }\end{array}$ \\
\hline No statin use & $1.00($ reference $)$ & $1.00($ reference $)$ \\
\hline Low potency statin & $1.24(1.22-1.26)$ & $1.21(1.19-1.24)$ \\
\hline Medium potency & $1.26(1.25-1.27)$ & $1.22(1.21-1.23)$ \\
\hline High potency statin & $1.39(1.38-1.41)$ & $1.34(1.32-1.36)$ \\
\hline P linear trend & $<0.0001$ & $<0.0001$ \\
\hline
\end{tabular}

*Adjusted for age (continuous), sex, race, and BMI (continuous)

Table-2: Hazard ratio (95\% CI) for Type 2 diabetes according to statin potency in US Veterans

\section{Association of statin treatment with plasma glucose over time}

Among 273,970 Veterans who were prescribed statin and were eligible for analysis of pre- and post-statin plasma glucose, we observed comparable average random plasma glucose before and after statin initiation. Mean values of random plasma glucose 12 months before and 12 months after statin initiation were $108.9(\mathrm{SD}=37.5)$ and 108.9 $(\mathrm{SD}=34.5) \mathrm{mg} / \mathrm{dl}$, respectively, $\mathrm{p}$ difference 0.92

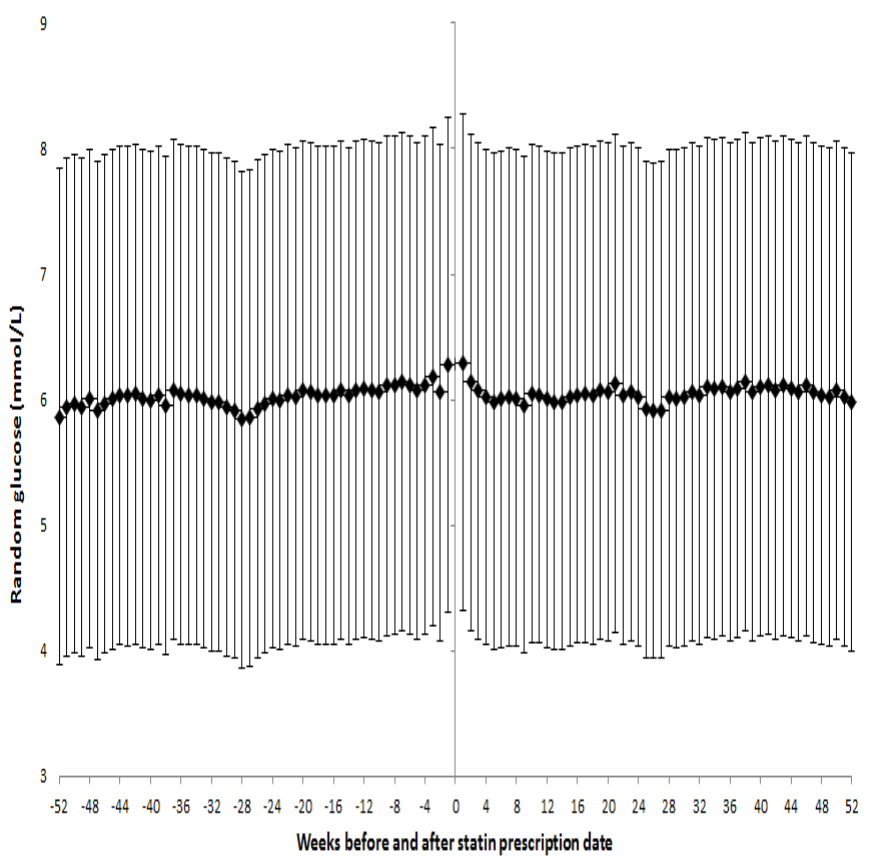

Figure- 2 Mean glucose (95\% confidence interval) 52 weeks before and 52 weeks after the first filling of statin prescription among US Veterans.

\section{Discussion}

In this large prospective cohort of US Veterans, we showed that statin use was associated with a higher risk of Type 2 diabetes and such relation was graded according to statin potency. The positive relation between statin use and incidence of Type 2 diabetes was seen in both men and women and was not modified by race, body mass index or triglycerides. 
In our data, we observed a higher risk of Type 2 diabetes among users of statin. This observation is consistent with another observational study that reported a $46 \%$ higher risk of Type 2 diabetes (95\% CI: $22 \%$ to $74 \%$ ) in 8,749 subjects after an average follow up of 5.9 years [18]. Furthermore, our data are in line with JUPITER trial [19], where treatment with rosuvastatin increased the risk of Type 2 diabetes by $28 \%$ compared to placebo (95\% CI: $7 \%$ to $54 \%$ ) among subjects with at least one risk factor for developing diabetes (metabolic syndrome, impaired fasting glucose, obese, or hemoglobin A1C $>6 \%$ ). Of note is that the increased risk of Type 2 diabetes was not observed in JUPITER subjects free of Type 2 diabetes risk factors [19],. In a meta-analysis of five randomized trials with 32,752 subjects and 2,749 Type 2 diabetes cases, Preiss et al. [20], reported a $12 \%$ increased odds of Type 2 diabetes with statin compared to placebo (95\% CI: $4 \%$ to $22 \%$ ). In a recent metaanalysis of 25 randomized trials including 141,863 subjects, the pooled relative risk for Type 2 diabetes was 1.12 (95\% CI: 1.05-1.21) [17]. In contrast, an earlier and smaller meta-analysis of 17 randomized trials with 113,394 subjects did not show an association between statin use and incidence of Type 2 diabetes [pooled relative risk of 1.15 (95\% CI: $0.90-1.50)^{21}$ ]. Of note is that the effect size was consistent with modest elevated risk of Type 2 diabetes and the lack of statistical significance was probably due to limited statistical power. In addition, other smaller studies failed to show elevated risk of Type 2 diabetes with statin use $[9,10,12,22]$. It is possible that null findings reported in those studies were a consequence of inadequate statistical power. Alternatively, heterogeneity of Type 2 diabetes phenotype and resulting variability in response to statin treatment in those populations could be another explanation. In a cluster analysis, Ahlqvist and colleagues [23,] showed that subjects with Type 2 diabetes characterized by severe insulin resistance and high body mass index had serious diabetic complications than people with Type 2 diabetes caused by autoimmune disorder or other Type 2 diabetes phenotypes characterized by absence of insulin resistance or adiposity. The anti-inflammatory effects of statin could mitigate the risk of developing Type 2 diabetes caused by chronic inflammation as would be the case in adiposity and insulin resistance. For now, we can only speculate on these hypotheses in the absence of welldesigned clinical and epidemiologic studies focused on these hypotheses.

Our study showed a graded response between statin potency (dose) and incidence of Type 2 diabetes as reported by others [18]. This suggests that the risk of Type 2 diabetes could be minimized with lower dose of statin, especially in patients at risk of Type 2 diabetes.
In contrast, our secondary analysis did not show any meaningful elevation in random plasma glucose after statin initiation in this cohort of Veterans. Our results are consistent with data from the JUPITER trial [19], where an intervention with $20 \mathrm{mg} / \mathrm{d}$ of rosuvastatin did not increase fasting glucose at 24 months of follow up (median glucose $98 \mathrm{mg} / \mathrm{dl}$ ) compared to placebo (median glucose $98 \mathrm{mg} / \mathrm{dl}$ ) in 17,802 healthy adults ${ }^{4}$. Furthermore, in a trial of 146 dyslipidemic patients, a 12-month intervention with $1 \mathrm{mg} / \mathrm{d}$ of pitavastatin resulted in a $3 \mathrm{mg} / \mathrm{dl}$ reduction in fasting plasma glucose compared to placebo[24]. In contrast, other studies reported elevated glucose concentration after statin initiation. In a randomized trial of 146 hypercholesterolemia subjects, $5 \mathrm{mg} / \mathrm{d}$ of atorvastatin resulted in a $3 \mathrm{mg} / \mathrm{dl}$ increase in fasting serum glucose compared to placebo after 12 months $(p<0.01)$ [24]. In addition, atorvastatin intervention increased serum insulin and homeostatic model of insulin resistance compared to placebo[24]. In another trial of 29 people with Type 2 diabetes and dyslipidemia, an intervention with simvastatin $(10 \mathrm{mg} / \mathrm{d})$ led to $18 \%$ increase in fasting glucose compared with baseline value after 4 months of intervention [25]. The apparent discrepancy between these trials and our observational findings merits some comments. First, we did not have fasting glucose in our study for appropriate comparison with trial data mentioned above. Second, heterogeneity in study populations and Type 2 diabetes phenotype [23], could partly explained the discrepancy (VA population was mostly male and free of Type 2 diabetes while JUPITER [25], recruited subjects with elevated C-reactive protein and other trials recruited Type 2 diabetes subjects [3] or older adults with dyslipidemia (mean age 65 years) [24] vs. 60 years in our study.

What potential mechanisms might support causal effect of statin on Type 2 diabetes incidence?

- Several potential biologic mechanisms have been proposed including inhibition of glucose uptake [26].

Studies have demonstrated that simvastatin can rapidly and reversibly block insulin secretion in single-islet cultures [27] and cell cultures [28]. In another experiment using L6 myotubules, both atorvastatin and simvastatin inhibited insulin-mediated glucose uptake by blocking insulin receptor substrate-1 (IRS-1) phosphorylation [18]. Statin may also inhibit glucose uptake via increase in cellular non-esterified fatty acid concentration; statin treatment has been shown to induce expression of fatty acid synthase and acetyl coA carboxylase 1 [29] and increase in free fatty acids [30]. Treatment of cultured rat cardiomyocytes with atorvastatin led to reduction in glucose uptake and reduction in mRNA and protein expression of glucose transporter type 4 [31,32] and IRS-1 [32]. Another hypothesis is that statin might heighten the risk of Type 2 diabetes by increasing insulin resistance $[33,34]$. 
The current study has some limitations including the use of electronic health records to ascertain exposure and outcome. Due to a lack of information on hemoglobin A1C and fasting glucose, we were not able to use the American Diabetes Association definition for incident Type 2 diabetes. The observational design of our study does not preclude unmeasured confounding as alternative explanation of observed results. Although 94\% of Veterans were male, we had data on nearly 223,000 women to show a similar relation.

Despite above limitations, our study has numerous strengths including a large sample size; availability of data on various statin brands and several racial groups across the entire US; and the robustness of findings in secondary analyses. Given the efficacy of statin in the prevention of cardiovascular disease, the reported modest elevation of Type 2 diabetes risk with statin use does not affect the utility of statins in preventive cardiology. Our findings suggest that in the presence of strong family history of Type 2 diabetes or other risk factors for Type 2 diabetes such as obesity, clinicians might consider low-potency statin supplemented by other drugs (non-statin) or modifiable lifestyle factors for management of dyslipidemia. The availability of PCSK9 drugs may also be considered when subjects being evaluated for statin treatment are at higher risk of developing Type 2 diabetes.

In conclusion, our findings show a positive association between statin use and incidence of Type 2 diabetes among US Veterans.

\section{Acknowledgements:}

We also thank Mrs. Constance Nelson, MPH for her help with logistics and administrative support. Dr. Djousse has full access to data used for this manuscript.

Data availability: Drs. Djousse, Gagnon, and Cho have full access to the data sets. As per the Department of Veterans Affairs' policy, veterans data are not available to the public.

Source of funding: This study was supported by investigator-initiated grant from Merck (to LD). The study sponsor was not involved in the design of the study; the collection, analysis, and interpretation of data; writing the report; or the decision to submit the report for publication. No other disclosures from the authors.

Funding source: Supported by an investigatorinitiated grant from Merck (Protocol \# 2922).

\section{Contribution statement:}

- Substantial contributions to conception and design, acquisition of data or analysis and interpretation of data: Djousse, Song, Cho, Gaziano, and Gagnon.
- Drafting the article or revising it critically for important intellectual content: Djousse, Song, Cho, Gaziano, and Gagnon.

- Final approval of the version to be published: Djousse, Song, Cho, Gaziano, and Gagnon.

\section{Conflicts of Interest: None.}

\section{References}

1. Egan BM, Li J, White K, et al. 2013 ACC/AHA Cholesterol Guideline and Implications for Healthy People 2020 Cardiovascular Disease Prevention Goals. J Am Heart Assoc 2016; 5.

2. Pletcher MJ, Pignone M, Jarmul JA, Moran AE, Vittinghoff E, Newman T. Population Impact \& Efficiency of Benefit-Targeted Versus Risk-Targeted Statin Prescribing for Primary Prevention of Cardiovascular Disease. J Am Heart Assoc 2017;6.

3. Gonzalez-Clemente JM, Palma S, Arroyo J, et al. [Is diabetes mellitus a coronary heart disease equivalent? Results of a meta-analysis of prospective studies]. Rev Esp Cardiol 2007; 60:1167-76.

4. Juutilainen A, Lehto S, Ronnemaa T, Pyorala K, Laakso M. Type 2 diabetes as a "coronary heart disease equivalent": an 18-year prospective population-based study in Finnish subjects. Diabetes Care 2005;28:2901-7.

5. Kannel WB, Dawber TR, Friedman GD, Glennon WE, McNamara PM. Risk factors in coronary heart disease; an evaluation of several serum lipids as predictors of coronary heart disease. AnnInternMed 1964;61:888-99.

6. Jackson CL, Wee CC, Hurtado DA, Kawachi I. Obesity trends by industry of employment in the United States, 2004 to 2011. BMC Obes 2016;3:20.

7. Jackson CL, Szklo M, Yeh HC, et al. Black-white disparities in overweight and obesity trends by educational attainment in the United States, 19972008. J Obes 2013;2013:140743.

8. Wild S, Roglic G, Green A, Sicree R, King H. Global prevalence of diabetes: estimates for the year 2000 and projections for 2030. Diabetes Care 2004;27:1047-53.

9. Freeman DJ, Norrie J, Sattar N, et al. Pravastatin and the development of diabetes mellitus: evidence for a protective treatment effect in the West of Scotland Coronary Prevention Study. Circulation 2001;103:357-62.

10. Collins R, Armitage J, Parish S, Sleigh P, Peto R, Group HPSC. MRC/BHF Heart Protection Study of cholesterol-lowering with simvastatin in 5963 people with diabetes: a randomised placebo-controlled trial. Lancet 2003;361:2005-16.

11. Sever PS, Dahlof B, Poulter NR, et al. Prevention of coronary and stroke events with atorvastatin in hypertensive patients who have average or lowerthan-average cholesterol concentrations, in the 
Anglo-Scandinavian Cardiac Outcomes Trial-Lipid Lowering Arm (ASCOT-LLA): a multicentrerandomised controlled trial. Drugs 2004;64 Suppl 2:43-60.

12. Keech A, Colquhoun D, Best J, et al. Secondary prevention of cardiovascular events with long-term pravastatin in patients with diabetes or impaired fasting glucose: results from the LIPID trial. Diabetes Care 2003;26:2713-21.

13. Ridker PM, Danielson E, Fonseca FA, et al. Rosuvastatin to prevent vascular events in men and women with elevated C-reactive protein. N Engl J Med 2008;359:2195-207.

14. Shepherd J, Blauw GJ, Murphy MB, et al. Pravastatin in elderly individuals at risk of vascular disease (PROSPER): a randomised controlled trial. Lancet 2002;360:1623-30.

15. Shen L, Shah BR, Reyes EM, et al. Role of diuretics, beta blockers, and statins in increasing the risk of diabetes in patients with impaired glucose tolerance: reanalysis of data from the NAVIGATOR study. BMJ 2013;347:f6745.

16. Rahal AJ, ElMallah AI, Poushuju RJ, Itani R. Do statins really cause diabetes? A meta-analysis of major randomized controlled clinical trials. Saudi Med J 2016;37:1051-60.

17. Thakker D, Nair S, Pagada A, Jamdade V, Malik A. Statin use and the risk of developing diabetes: a network meta-analysis. Pharmacoepidemiol Drug Saf 2016;25:1131-1149.

18. Cederberg H, Stancakova A, Yaluri N, Modi S, Kuusisto J, Laakso M. Increased risk of diabetes with statin treatment is associated with impaired insulin sensitivity and insulin secretion: a 6 year follow-up study of the METSIM cohort. Diabetologia 2015;58:1109-17.

19. Ridker PM, Pradhan A, Macfadyen JG, Libby P, Glynn RJ. Cardiovascular benefits and diabetes risks of statin therapy in primary prevention: an analysis from the JUPITER trial. Lancet 2012;380:565-71.

20. Preiss D, Seshasai SR, Welsh P, et al. Risk of incident diabetes with intensive-dose compared with moderate-dose statin therapy: a metaanalysis. JAMA 2011;305:2556-64.

21. Navarese EP, Buffon A, Andreotti F, et al. Metaanalysis of impact of different types and doses of statins on new-onset diabetes mellitus. Am J Cardiol 2013;111:1123-30.

22. Downs JR, Clearfield M, Weis S, et al. Primary prevention of acute coronary events with lovastatin in men and women with average cholesterol levels: results of AFCAPS/TexCAPS. Air Force/Texas Coronary Atherosclerosis Prevention Study. JAMA 1998;279:1615-22.
23. Ahlqvist E, Storm P, Karajamaki A, et al. Novel subgroups of adult-onset diabetes and their association with outcomes: a data-driven cluster analysis of six variables. Lancet Diabetes Endocrinol 2018;6:361-9.

24. Nakagomi A, Shibui T, Kohashi K, et al. Differential Effects of Atorvastatin and Pitavastatin on Inflammation, Insulin Resistance, and the Carotid Intima-Media Thickness in Patients with Dyslipidemia. J AtherosclerThromb 2015;22:115871.

25. Ohrvall M, Lithell H, Johansson J, Vessby B. A comparison between the effects of gemfibrozil and simvastatin on insulin sensitivity in patients with non-insulin-dependent diabetes mellitus and hyperlipoproteinemia. Metabolism 1995;44:212-7.

26. Nowis D, Malenda A, Furs K, et al. Statins impair glucose uptake in human cells. BMJ OpenDiabetes ResCare 2014;2:e000017.

27. Scattolini V, Luni C, Zambon A, et al. Simvastatin Rapidly and Reversibly Inhibits Insulin Secretion in Intact Single-Islet Cultures. Diabetes Ther 2016;7:679-93.

28. Yaluri N, Modi S, Lopez RM, et al. Simvastatin Impairs Insulin Secretion by Multiple Mechanisms in MIN6 Cells. PLoSOne 2015;10:e0142902.

29. Brown MS, Goldstein JL. The SREBP pathway: regulation of cholesterol metabolism by proteolysis of a membrane-bound transcription factor. Cell 1997;89:331-40.

30. Kain V, Kapadia B, Misra P, Saxena U. Simvastatin may induce insulin resistance through a novel fatty acid mediated cholesterol independent mechanism. Sci Rep 2015;5:13823.

31. Nakata M, Nagasaka S, Kusaka I, Matsuoka H, Ishibashi S, Yada T. Effects of statins on the adipocyte maturation and expression of glucose transporter 4 (SLC2A4): implications in glycaemic control. Diabetologia 2006;49:1881-92.

32. Jiang Z, Yu B, Li Y. Effect of Three Statins on Glucose Uptake of Cardiomyocytes and its Mechanism. MedSci Monit 2016;22:2825-30.

33. Aiman U, Najmi A, Khan RA. Statin induced diabetes and its clinicalimplications. J PharmacolPharmacother 2014;5:181-5.

34. Dahagam C, Hahn VS, Goud A, et al. Role of statins in glucose homeostasis and insulin resistance. Curr Cardiovasc Risk Rep 2016;10:40. 